\title{
Netflix na Disciplina Auditoria de Sistemas: Um Relato de Aplicação de Aprendizagem Ativa
}

\author{
Ronney Moreira Castro ${ }^{1}$ e Tadeu Moreira de Classe $^{1}$ \\ ${ }^{1}$ Programa de Pós-Graduação em Informática (PPGI) \\ Universidade Federal do Rio Estado do Rio de Janeiro (UNIRIO) \\ Rio de Janeiro - RJ - Brasil \\ \{ronney.castro, tadeu.classe\}@uniriotec.br
}

\begin{abstract}
Systems audit is a discipline from the computer science field that is mainly theoretical. Thus, they have become more expositive in remote classes because of the COVID-19 pandemic. However, active learning techniques can support these classes, building a scenario where the students can think and discuss subjects among themselves. This article presents a report of the application of the flipped classroom technique, which the students watched the Netflix's TV show, Onisciente to look for organizational security failures and build a risk matrix. The activity allowed the students to catch a glimpse of a day into an organization, being the own auditors like in a real scenario. Thus, the students could identify elements to compose the risk matrix from the Onisciente enterprise. Also, they answered their perceptions about the activity and they said that it was a different learning way, more pleasant and hard at the same time and, it was a creative manner to engage students in their learning and teaching process.
\end{abstract}

Resumo. Auditoria de sistemas é uma disciplina da área de computação predominantemente teórica, a qual, devido às aulas remotas da COVID19, se tornou ainda mais expositiva. Neste contexto, o uso de técnicas de Aprendizagem Ativa pode auxiliar nas aulas, criando meios em que os próprios alunos reflitam e discutam conteúdos entre si. Este artigo apresenta um relato de aplicação da técnica de sala de aula invertida, no qual os alunos, ao assistirem a série Onisciente da Netflix, buscaram identificar as falhas de segurança organizacional, construindo matrizes de risco auditando a empresa. A atividade permitiu aos alunos terem um vislumbre do dia-a-dia de uma empresa, sendo eles os próprios auditores, como em um caso de uso real. Assim, conseguiram identificar elementos para compor a matriz de risco da empresa Onisciente. Ao serem questionados sobre a atividade relataram que a atividade foi uma forma diferente de aprender, sendo prazerosa e desafiadora ao mesmo tempo, além de trazer um meio criativo de engajar os alunos em seu processo de ensino e aprendizagem.

\section{Introdução}

Os cursos de graduação da área da computação são compostos por disciplinas que abordam diversos conteúdos, como: algoritmos, estrutura de dados, banco de dados, orientação a objetos, engenharia de software e outros. Uma disciplina que está presente na 
maioria deles é a "Auditoria de Sistemas", que aborda a verificação de conformidades nos sistemas, se padrões, normas e políticas estão sendo devidamente seguidos e, até mesmo, englobando assuntos de outras disciplinas, como, por exemplo, redes de computadores [Imoniana 2016].

O modelo de construção das ementas e ensino das disciplinas segue o paradigma de orientação ao conteúdo que deve ser assimilado pelos alunos. Dessa forma, o professor passa a figurar como o detentor e disseminador do conhecimento [Araújo et al. 2015]. Em disciplinas que possuem conteúdo predominantemente teórico, como é o caso de "Auditoria de Sistemas", os discentes ficam focados em absorver as temáticas, não permitindo a eles associá-los à prática que o mercado demanda. No mundo contemporâneo o pensamento crítico, a colaboração, a capacidade de solucionar problemas e a tomada de decisões são habilidades fundamentais que os discentes devem desenvolver para enfrentar os desafios do mercado [Acosta et al. 2016].

No geral, o modelo de aula mais utilizado por professores é o expositivo. Isso deve-se muito ao fato dos docentes, durante toda a sua formação, terem convivido com professores que usavam tal abordagem e acabam por replicar as mesmas práticas que aprenderam [Castro e Siqueira 2019b]. Este modelo pedagógico, vem se tornando obsoleto com o passar dos anos. Na antiga sociedade industrial, onde havia a produção em massa de estudantes, o professor era o transmissor do conhecimento, porém, cada vez mais o mundo vem mudando e este modelo tornando-se deficitário em relação às gerações de estudantes que estão buscando sua colocação na atual economia global [Tapscott e Williams 2010]. Isso implica em buscar alternativas educacionais que estejam alinhadas ao novo estilo de aprendizagem dos alunos [Kessler et al. 2010].

Várias estratégias vêm se destacando para colocar os discentes dessa geração como protagonistas nas aulas, e inovando nas formas de ensino e aprendizagem. Exemplo destas estratégias podem ser citados: aprendizagem baseada em problemas, dinâmicas, sala de aula invertida, dentre outras [Castro e Siqueira 2019b]. Essas abordagens estão diretamente relacionadas à Aprendizagem Ativa (AA). As técnicas de AA permitem, que os alunos sejam mais imersos em atividades dentro e fora da sala de aula e, além disso, saiam de seu papel de apenas ouvintes [Massey et al. 2005].

Desta maneira, este trabalho tem o objetivo de trazer um relato de uma experiência na utilização da técnica de "Sala de Aula Invertida", a partir do uso do seriado "Onisciente ${ }^{1}$ " da Netflix ${ }^{2}$, na disciplina de "Auditoria de Sistemas" durante o segundo semestre de 2020. Este período envolveu o uso de aulas remotas por conta da pandemia de COVID-19 e o uso deste seriado visou colocar os alunos como auditores dentro de uma empresa e detectar suas vulnerabilidades, temas constantes observados na temática de auditoria de sistemas e que são essenciais no mercado de sistemas de informação.

A organização deste artigo traz na Seção 2 os conceitos fundamentais do trabalho. A Seção 2.3 apresenta uma breve visão do ensino durante a pandemia. A Sseção 3 aborda a atividade desenvolvida com os alunos. E, finalmente, a Seção 4 traz uma breve discussão e principais conclusões.

\footnotetext{
${ }^{1}$ Onisciente: https://www.netflix.com/br/title/80220334

${ }^{2}$ Netflix: https://www.netflix.com
} 


\section{Conceitos Fundamentais}

\subsection{Aprendizagem Ativa}

A sociedade moderna pode ser considerada digital principalmente por conta do avanço e da proliferação de diversas tecnologias como a computação e a comunicação. A internet, os smartphones e outros gadgets já ocupam papel de destaque no cotidiano das pessoas. Com essa evolução, o perfil e o processo de aprendizado dos alunos foram alterados [Loureiro e Messias 2016]. Se tratando do mercado da Tecnologia da Informação, as empresas buscam pessoal qualificado com habilidades técnicas e analíticas [Bradley et al. 2005]. Preparar os alunos para serem qualificados para este mercado exige proporcionar uma educação que transmita, melhore e desenvolva suas competências/habilidades na área [Larkin-Hein e Zollman 2000].

Diante desse cenário, o uso da Aprendizagem Ativa (AA) pode auxiliar de forma significativa. A AA pode ser definida como "atividades instrucionais envolvendo os alunos a fazerem coisas e pensar sobre o que estão fazendo" [Bonwell e Eison 1991, Massey et al. 2005]. As técnicas de AA permitem, não somente que os alunos participem de atividades dentro e fora da sala de aula, mas também saiam de seu papel de apenas ouvintes com o professor sendo o centro das atenções. [Mitchell et al. 2017] atualizaram esse conceito e afirmam que a AA são quaisquer exercícios introduzidos na aula para estimular o pensamento e a participação dos alunos em um esforço para envolvê-los no processo de aprendizagem.

Um grande número de estratégias de AA vem ganhando destaque, na promessa de obter a atenção dos alunos e auxiliá-los na aprendizagem, tais como salas de aula invertida [Martins e Gouveia 2019], uso de dinâmicas de grupo [Castro e Siqueira 2018], jogos [Classe e Castro 2020], entre outras. O portal AlcaSystem ${ }^{3}$ é uma ferramenta que permite a busca, seleção, classificação e recomendação de técnicas de AA extraídas de artigos em conferências e revistas nacionais sobre informática na educação (CBIE, SBIE, WEI, WIE e RBIE) e pode auxiliar docentes que queiram introduzir técnicas AA em suas aulas [Castro e Siqueira 2019a].

\subsection{Auditoria de Sistemas}

Os sistemas estão em constante evolução e tornam-se cada vez mais sofisticados, assumindo um papel de destaque no cotidiano das pessoas e das empresas. A globalização e os avanços tecnológicos na área da tecnologia da informação, intensificaram a busca por informação por toda a sociedade. Grandes corporações não mais sobrevivem sem o uso de um sistema. Diante disso, surgiram muitos problemas como falhas, lentidão, vulnerabilidades, entre outros [Imoniana 2016].

Com o objetivo de assegurar a integridade e confiabilidade das informações, tornou-se necessário a criação de medidas de controle e segurança dos sistemas de informação. A Auditoria de Sistemas de Informação tem a função de avaliar e validar os resultados gerados pelos Sistemas de Informação, verificar a eficiência dos processos concluídos, além de verificar a segurança e confiabilidade das informações [Attie 2006].

A disciplina de Auditoria de Sistemas é lecionada, em geral, nos últimos períodos dos cursos de graduação da área da Computação, principalmente porque os discentes

\footnotetext{
${ }^{3}$ AlcaSystem: http://alcasystem.uniriotec.br
} 
terão conhecimentos sobre vários conceitos importantes relacionados ao desenvolvimento de um sistema de informação, tais como: Análise e Desenvolvimento de Sistemas, Engenharia de Software, Redes de Computadores, etc. A disciplina busca apresentar conceitos fundamentais aplicados em uma disciplina de Auditoria de Sistemas de Informação focando nas etapas para sua a realização e também nos controles que devem ser verificados em um processo real de trabalho.

\subsection{O Ensino no Período de Pandemia COVID-19}

Em dezembro de 2019, foi descoberto na cidade de Wuhan na China, um novo vírus conhecido por Coronavírus 2 da síndrome respiratória aguda grave (SARS-CoV-2). A partir desse momento, o vírus começou a se espalhar por diversos países e a Organização Mundial de Saúde (OMS) declarou emergência de saúde pública internacional em 30 de janeiro de 2020 e a uma pandemia em 11 de março de 2020, conhecida por Pandemia do COVID-19 [Huang et al. 2020]. Diante desse quadro, diversos países decidiram fechar escolas, faculdades e universidades com o objetivo de reduzir o contato entre os indivíduos e, com isso, preservar a vida.

Com a pandemia, as Instituição de Ensino Superior (IES) em todo o mundo, foram obrigadas a mudar sua forma de trabalho e, consequentemente, utilizar alternativas para levar o aprendizado aos alunos. Isso inclui desde o uso simples de correspondências com conteúdo para os alunos, até o uso de ferramentas tecnológicas que propiciam um contato remoto entre professor e aluno. Por outro lado, os docentes também tiveram que alterar/adaptar seus processos de ensino, experimentando novas técnicas, ferramentas, entre outros. [Burgess e Sievertsen 2020, Luthra e Mackenzie 2020].

No Brasil, a situação não foi diferente. O Ministério da Educação (MEC) se manifestou, através da Portaria $n^{\circ} 343$ de 17/03/2020, sobre a substituição das aulas presencias por aulas usando meios digitais, enquanto perdurar o cenário de pandemia COVID-19 [Brasil 2020]. Diante disso, o Ensino Remoto Emergencial (ERE) assumiu o papel das aulas presenciais, como forma de mitigar os impactos na aprendizagem dos alunos O ERE é diferente do Ensino a Distância (EaD), uma modalidade de ensino antiga no país que possui legislação específica. O ERE foi regulamentado pela Portaria $n^{\circ} 343$, com o objetivo de atender à situação específica causada pela pandemia [Goedert e Arndt 2020].

Diante desse cenário, o ano de 2020 foi marcado pelas aulas remotas. Isso impediu o contato presencial entre professor e aluno, e também restringiu o uso de certas técnicas de AA, que seriam mais facilmente aplicadas em sala de aula presencial como, por exemplo, as dinâmicas de grupo.

\section{Uso de Aprendizagem Ativa no Ensino de Auditoria de Sistemas}

A disciplina Auditoria de Sistemas faz parte da grade do Curso de Tecnólogo em Análise e Desenvolvimento de Sistemas (ADS) de uma IES na cidade de Juiz de Fora (MG), em seu último período. O material de apoio utilizado pelos docentes é composto apenas por apresentações de slides e alguns exercícios para cada capítulo. Assim, o conteúdo é predominantemente expositivo.

São vários os conteúdos definidos na ementa, como: conceitos de auditoria de sistema, ferramentas, tipos de auditoria, segurança de informação etc. Dentre eles, um 
conteúdo com grande relevância refere-se à Matriz de Risco (escore de risco), a qual deve ser considerada na fase de planejamento de uma auditoria. Essa matriz propicia uma visão geral de itens relevantes de uma empresa e permite ao auditor verificar quais deles possuem maior risco. Um exemplo de classificação da Matriz quanto ao impacto e probabilidade pode ser visualizado nas Tabelas 1 e 2, respectivamente, um exemplo do método de ponderação na Tabela 3, e um pequeno exemplo de itens em uma Matriz de Risco na Tabela 4.

Tabela 1. Classificação do Impacto para a Matriz de Risco

\begin{tabular}{|c|l|}
\hline Escala & Classificação do Impacto \\
\hline 0 & Impacto irrelevante. \\
\hline 1 & Efeito pouco significativo, sem afetar a maioria dos processos da empresa. \\
\hline 2 & $\begin{array}{l}\text { Sistemas indisponíveis por um determinado período de tempo, podendo causar perda de } \\
\text { credibilidade e perdas financeiras. }\end{array}$ \\
\hline 3 & Perdas financeiras mais significativas e perda de clientes para concorrentes. \\
\hline 4 & Efeitos desastrosos, mas que não comprometam a sobrevivência da organização. \\
\hline 5 & Efeitos desastrosos que comprometam a sobrevivência da organização. \\
\hline
\end{tabular}

Tabela 2. Classificação da Probabilidade para a Matriz de Risco

\begin{tabular}{|c|l|}
\hline Escala & Classificação do Impacto \\
\hline 0 & Ameaça completamente improvável de acontecer. \\
\hline 1 & Probabilidade da ameaça ocorrer menos de uma vez por ano. \\
\hline 2 & Probabilidade da ameaça ocorrer pelo menos uma vez por ano. \\
\hline 3 & Probabilidade da ameaça ocorrer pelo menos uma vez por mês. \\
\hline 4 & Probabilidade da ameaça ocorrer pelo menos uma vez por semana. \\
\hline 5 & Probabilidade da ameaça ocorrer diariamente. \\
\hline
\end{tabular}

Tabela 3. Ponderação para a Matriz de Risco

\begin{tabular}{|c|l|}
\hline Score de Risco & Prioridade \\
\hline $0-5$ & Muito Baixa \\
\hline $6-10$ & Baixa. \\
\hline $11-15$ & Média \\
\hline $16-20$ & Alta \\
\hline $21-25$ & Muito Alta \\
\hline
\end{tabular}

Tabela 4. Exemplo de uma parte de uma Matriz de Risco

\begin{tabular}{|l|c|c|l|c|}
\hline Ameaças & Impacto (I) & Probabilidade (P) & $\begin{array}{c}\text { Escore de } \\
\text { Risco (I x P) }\end{array}$ & Prioridade \\
\hline Incêndio & 5 & 1 & 5 & Muito Baixa \\
\hline Indisponibilidade de servidor & 3 & 3 & 9 & Baixa \\
\hline Funcionário que adoece & 1 & 1 & 1 & Muito Baixa \\
\hline Ataque de vírus & 3 & 5 & 15 & Média \\
\hline Falha de switch de rede & 2 & 4 & 8 & Baixa \\
\hline
\end{tabular}

No geral, ao lecionar tais conteúdos, é necessário um estudo de caso em uma organização sendo essa fictícia ou não. Para ilustrar e fazer com que os alunos pudessem construir uma Matriz de Risco baseada em algum exemplo de uma organização, o professor responsável pela disciplina Auditora de Sistemas resolveu implantar no segundo 
semestre de 2020 uma técnica de AA, a sala de aula invertida (também conhecida por "flipped classroom"), mudando a forma expositiva que a disciplina é normalmente contemplada. Essa técnica preconiza que a parte teórica deve ser estudada pelos alunos antes da aula e, no dia da aula, os discentes devem trazer suas dúvidas, além do conteúdo ser aprofundado através de exercícios, discussões, mapas mentais e mecanismos didáticos. Nesse modelo o docente torna-se um mediador, estimulador, norteador [Zainuddin e Halili 2016].

\subsection{Netflix e a Série Onisciente}

A Netflix é uma plataforma de streaming muito popular no mundo inteiro. No ano de 2020 obteve a marca de 200 milhões de assinantes, principalmente por conta da diversidade, conteúdo fornecido, a excelente qualidade do serviço e o preço reduzido [Alexander 2020]. Com base nisso, é muito comum os alunos possuírem interesse nessa plataforma. No caso específico da IES e da turma de ADS do segundo semestre de 2020, todos os discentes possuíam acesso.

Uma das series brasileiras da plataforma é a Onisciente. A história se passa no futuro, na cidade de São Paulo, onde cada cidadão é monitorado $24 \mathrm{~h}$ por dia por um drone individual, que avalia as atitudes através de um sistema de Inteligência Artificial denunciando possíveis infrações. Tal sistema tem o nome de Onisciente é mantido pela Empresa de mesmo nome e tendo objetivo de levar a cidade a criminalidade zero. A personagem principal, Nina, participa de um programa de trainees dentro da Empresa, mas ao chegar em casa e ver o pai morto, elabora um plano para conseguir acesso às imagens do drone do pai e, consequentemente, desvendar este crime. Para isso, ela precisa entrar em partes específicas da Onisciente, invadir o sistema, acessar servidores com segurança máxima, entre outros. Isso mostra que Nina deverá explorar vulnerabilidades na Empresa para alcançar seu objetivo.

\subsection{Matriz de Risco da Empresa Onisciente}

Baseando-se nisso, o professor solicitou que os alunos assistissem a série (6 episódios), verificando quais as vulnerabilidades que a personagem Nina ia explorando dentro da empresa. Além disso, ele pediu para que as vulnerabilidades fossem inseridas em uma Matriz de Risco, calculando a pontuação deles por cada um dos discentes. Para a tarefa foi dado um prazo de 15 dias. Ao término, os alunos deveriam levar para a aula suas matrizes e também considerações que achavam importantes a respeito do conteúdo visto na disciplina e o que puderam perceber deste em relação à série.

Por conta da limitação de espaço não é possível mostrar todas as matrizes da turma. Optou-se por exibir uma delas na Tabela 5 (parte da matriz gerada por um dos discentes). O professor também aproveitou para solicitar aos alunos que colocassem a atividade de controle de determinado risco e algum comentário relevante. Tais atividades fazem parte da auditoria e, é através delas que o auditor determina o risco. Os controles compreendem políticas, processos e procedimentos [Imoniana 2016].

No dia da aula, o professor pediu aos alunos que mostrassem suas matrizes aos demais colegas e fossem falando o que identificaram. A discussão foi muito promissora, tendo em vista que os alunos encontraram ameaças em comum e também distintas não identificadas por outros alunos. A pontuação de impacto e probabilidade também foi 
diferente, porém nada que mudasse consideravelmente o resultado final. Isso demonstra que não existe um padrão de análise, ou seja, existem visões diferentes para auditores (discentes) diferentes. Os alunos mesmo em ambiente remoto, por conta da pandemia, participaram de todo procedimento e acharam que a experiência foi muito válida. Não necessitaram sair de suas casas para "vivenciar" o cotidiano de uma Empresa e fazer a auditoria. Tudo foi feito baseado em uma série que contém conteúdo de Segurança da Informação e, consequentemente, de Auditoria de Sistemas.

Tabela 5. Parte de uma Matriz de Risco Elaborada por um dos Discentes

\begin{tabular}{|c|c|c|c|c|c|}
\hline Ameaças & Impacto & Probabilidade & Escore de Risco & Controles & Comentáro \\
\hline Queda de energia & 2 & 2 & 4 & $\begin{array}{l}\text { Apenas o } \\
\text { monitoramento } \\
\text { dos Drones }\end{array}$ & $\begin{array}{l}\text { Ao subir no telhado da empresa } \\
\text { onde trabalha, Nina se esconde } \\
\text { atrás de fios de eletricidade, } \\
\text { gerando um campo } \\
\text { eletromagnético sobre o seu } \\
\text { próprio drone e } \\
\text { consequentemente destruindo o } \\
\text { mesmo. Ela ganha um tempo } \\
\text { sem monitoramento de drone } \\
\text { até outro ser enviado. }\end{array}$ \\
\hline $\begin{array}{l}\text { Risco de pessoas não } \\
\text { autorizadas acessarem a sala. }\end{array}$ & 5 & 3 & 15 & $\begin{array}{l}\text { Um leitor de íris } \\
\text { que, ao identificar } \\
\text { um funcionário } \\
\text { válido, abre a } \\
\text { porta de acesso. } \\
\text { Monitoramento } \\
\text { dos Drones. } \\
\end{array}$ & $\begin{array}{c}\text { O leitor funciona } \\
\text { perfeitamente, porém, não é } \\
\text { adequado para receber a } \\
\text { entrada de um grupo de } \\
\text { funcionários. O Drone não } \\
\text { consegue controlar a restrição } \\
\text { de acesso de área }\end{array}$ \\
\hline $\begin{array}{l}\text { Risco de pessoas não } \\
\text { autorizadas acessarem a sala. }\end{array}$ & 4 & 4 & 16 & $\begin{array}{c}\text { Apenas o } \\
\text { monitoramento } \\
\text { dos Drones }\end{array}$ & $\begin{array}{c}\text { O Drone não monitora o acesso } \\
\text { do seu monitorado no que } \\
\text { tange a áreas restritas }\end{array}$ \\
\hline $\begin{array}{l}\text { Risco de pessoas sem drone } \\
\text { entrarem na cidade }\end{array}$ & 5 & 3 & 15 & $\begin{array}{l}\text { Apenas o } \\
\text { monitoramento } \\
\text { dos Drones }\end{array}$ & $\begin{array}{l}\text { Não existe uma proteção que } \\
\text { fica posicionada fixamente na } \\
\text { cidade, para auxiliar os drones } \\
\text { no monitoramento e o esgoto } \\
\text { não possui proteção nenhuma. }\end{array}$ \\
\hline $\begin{array}{l}\text { Ocorrer alterações de código- } \\
\text { fonte não registradas e } \\
\text { ausência de versionamento de } \\
\text { código. }\end{array}$ & 3 & 3 & 9 & $\begin{array}{l}\text { Apenas o } \\
\text { monitoramento } \\
\text { dos Drones }\end{array}$ & $\begin{array}{l}\text { Nem toda alteração realizada é } \\
\text { considerada uma atividade } \\
\text { suspeita, na visão dos Drones. } \\
\text { O Drone também não faz um } \\
\text { controle de restrição de acesso } \\
\text { a alguns terminais, permitindo } \\
\text { alterações realizadas nele. }\end{array}$ \\
\hline
\end{tabular}

\subsection{Avaliação da Atividade Onisciente}

Ao final da tarefa, o professor solicitou aos alunos que preenchessem um formulário contendo 5 questões discursivas. Assim, foi realizada uma análise qualitativa sobre as respostas. Apesar da turma e da quantidade de respostas ser muito pequena (12 alunos na turma) foi verificado um resultado positivos na percepção dos alunos. O objetivo será fazer uma análise mais criteriosa da atividade criada e comparar cenários diferentes.

A primeira questão: Sobre a Tarefa, na qual você deveria verificar as vulnerabilidades da Empresa Onisciente e construir a matriz de risco: $O$ que você aprendeu?, obteve indicações que os alunos conseguiram compreender o conteúdo envolvendo vulnerabilidades e também a construção de uma matriz de risco. Um dos respondentes comentou: "Em primeiro lugar aprendi a fazer a construção de uma matriz. de risco como dito, aprendi a olhar de uma nova forma para as empresas, com um olhar mais criterioso buscando suas falhas e vulnerabilidades, e mesmo não sendo presencial me senti muito imerso nesse conteúdo". 
A segunda questão: Sobre a Tarefa, na qual você deveria verificar as vulnerabilidades da Empresa Onisciente e construir a matriz de risco: $O$ que você acha que poderia melhorar? verificou se algo deveria ser modificado na tarefa. Os alunos responderam que a tarefa estava adequada ao que foi passado como conteúdo nas aulas. Na resposta de um deles: "Na minha opinião não há o que melhorar na forma de que foi passada a tarefa, achei muito boa a forma que foi administrada”.

Na terceira questão: Sobre a Tarefa, na qual você deveria verificar as vulnerabilidades da Empresa Onisciente e construir a matriz de risco: Qual o seu comentário final?, Percebe-se que eles gostaram do estilo diferente de atividade/aula, principalmente nessa resposta: "É um estilo de aula totalmente diferente do normal, e que agrega muito ao conhecimento do aluno, facilitando o aprendizado do mesmo". Outra resposta também mostra que a AA pode ser uma boa opção para os docentes em suas aulas: "Foi uma forma muito boa de aprender, uma forma de ensino que não fica restrita a perguntas e respostas como em provas normais, te leva a buscar aprender mais, e você se sente um profissional trabalhando, o que não acontece em uma prova normal”.

$\mathrm{Na}$ quarta questão: $O$ tipo de atividade foi diferente das tradicionais adotadas geralmente em sala de aula. Você acha que foi adequada a sua forma de aprender? Justifique., os alunos responderam que sim, principalmente porque a atividade tem significado. Mesmo usando uma série de ficção científica e não sendo uma empresa do mundo real, o conteúdo foi assimilado pelos discentes de forma significativa. Nas respostas de alguns alunos: "Super adequada, nos fez ter a visão real de como é trabalhar com auditoria de uma empresa."; "Sim, como disse na resposta anterior você aprende de uma forma mais dinâmica e melhor, ao meu modo de ver"; "Sim, como o trabalho envolveu uma série, nos motivou ainda mais a realizar esse trabalho.”.

A última questão: Neste período de Pandemia, com o ensino remoto, você acha que a atividade cumpriu o papel de análise da empresa Onisciente? Justifique., procurava saber dos alunos se a atividade, mesmo que no período de pandemia e com aulas remotas, atendeu seu objetivo. Os discentes responderam positivamente e uma das falas merece destaque: "Sim, neste momento que estamos vivendo acho que foi uma excelente alternativa, pois como não poderíamos estar dentro de uma empresa a ideia da série cumpriu muito bem as necessidade”. Ou seja, não seria fácil conseguir visitar uma empresa para fazer a atividade e o uso da série auxiliou muito nesse contexto.

No geral, a tarefa foi bem recebida pelos alunos, os quais conseguiram realizar o estudo de caso de uma empresa, "vivenciando" os problemas da Onisciente, conseguindo, de uma forma dinâmica e não expositiva, trabalhar com conceitos de segurança de informação na prática.

\section{Discussão Final e Conclusões}

A sociedade contemporânea vem se transformando ao longo dos anos. O rápido desenvolvimento das tecnologias de informação e comunicação tem mudado o perfil dos alunos. Além disso, torna-se fundamental para os discentes o pensamento crítico, a colaboração, a capacidade de solucionar problemas e a tomada de decisões.

As aulas tradicionais, totalmente expositivas, tendem a ser monótonas e desestimulantes, levando a um grande desinteresse por elas, ainda mais em tempos de 
pandemia e aulas remotas que vive-se atualmente. Diante desse contexto, a Aprendizagem Ativa (AA) pode auxiliar na melhoria do processo de ensino e aprendizagem.

Este trabalho apresentou um relato de uso de uma técnica de AA (sala de aula invertida), a partir da análise da série Onisciente da Netflix tendo como contexto a disciplina de Auditoria de Sistemas. O uso da técnica permitiu que os alunos analisassem a empresa como um estudo de caso e, assim, melhor assimilar do conteúdo das disciplina trazendo uma aprendizagem mais significativa, verificado, até mesmo, nas respostas dadas pelos alunos sobre a atividade realizada.

O uso de técnicas de AA não é a solução dos problemas de ensino e aprendizagem, mas pode contribuir efetivamente para aperfeiçoar a forma de ensino proporcionando aos alunos uma experiência inovadora e mais aliada a sua atual realidade de aprendizagem. Como trabalho futuro o professor pretende repetir a mesma tarefa em outras turmas e, até mesmo quando possível, em formato presencial.

\section{References}

Acosta, O., Reategui, E., e Behar, P. A. (2016). Recomendação de conteúdo em um ambiente colaborativo de aprendizagem baseada em projetos. In Congresso Brasileiro de Informática e Educação (CBIE).

Alexander, J. (2020). Netflix adds 15 million subscribers as people stream more than ever, but warns about tough road ahead. The Verge, 21.

Araújo, R., Santos, R., Farias, R. S., Franca, R., Silva, T., Vasconcelos, R., Tedesco, P., Padilha, M., e Belian, R. B. (2015). Investigação sobre inovações pedagógicas protagonizadas por docentes em uma instituição e ensino universitário no brasil. In CINDU2015-IV Congresso Internacional de Docência Universitária. Vigo. Anais do CINDU.

Attie, W. (2006). Auditoria interna: conceitos e aplicações. São Paulo: Atlas.

Bonwell, C. C. e Eison, J. A. (1991). Active learning: Creating excitement in the classroom. eric digest.

Bradley, R. V., Mbarika, V., Sankar, C. S., e Raju, P. (2005). Multimedia instructional materials in mis classrooms. In Encyclopedia of multimedia technology and networking, pages 717-723. IGI Global.

Brasil (2020). Portaria $\mathrm{n}^{\mathbf{0}}$ 343, de 17 de março de 2020. dispõe sobre a substituição das aulas presenciais por aulas em meios digitais enquanto durar a situação de pandemia do novo coronavírus - covid19. Disponível em: <http://www.in.gov.br/en/web/dou/-/ portaria-n-343-de-17-de-marco-de-2020-248564376>. Acessado em: 18 de maio de 2020.

Burgess, S. e Sievertsen, H. H. (2020). Schools, skills, and learning: The impact of covid-19 on education. VoxEu. org, 1.

Castro, R. M. e Siqueira, S. (2018). Desenvolvimento e avaliação de uma metodologia de aprendizagem ativa apoiada pelo uso de qr code para ensino de banco de dados. In Anais do XXVI Workshop sobre Educação em Computação. SBC. 
Castro, R. M. e Siqueira, S. (2019a). Alcasystem-um portal com técnicas de aprendizagem ativa para disciplinas da área da computação. In Anais dos Workshops do Congresso Brasileiro de Informática na Educação, volume 8, page 1243.

Castro, R. M. e Siqueira, S. (2019b). Metodologias, técnicas, ambientes e tecnologias alternativas utilizadas no ensino de algoritmos e programação no ensino superior no brasil. In Anais dos Workshops do Congresso Brasileiro de Informática na Educação, volume 8 , page 228 .

Classe, T. M. e Castro, R. M. (2020). Ludificando os fundamentos de computação através de aprendizagem ativa. In Anais do XXVIII Workshop sobre Educação em Computação, pages 116-120. SBC.

Goedert, L. e Arndt, K. B. F. (2020). Mediação pedagógica e educação mediada por tecnologias digitais em tempos de pandemia. Criar Educação, Criciúma, 9.

Huang, C., Wang, Y., Li, X., Ren, L., Zhao, J., Hu, Y., Zhang, L., Fan, G., Xu, J., Gu, X., et al. (2020). Clinical features of patients infected with 2019 novel coronavirus in wuhan, china. The lancet, 395(10223):497-506.

Imoniana, J. O. (2016). Auditoria de sistemas de informação . Grupo Gen-Atlas.

Kessler, M. C., de Paula, C. G., Albé, M. H., Manzini, N., Barcellos, C., Carlson, R., Marcon, D., e Kehl, C. (2010). Impulsionando a aprendizagem na universidade por meio de jogos educativos digitais. In Brazilian Symposium on Computers in Education (Simpósio Brasileiro de Informática na Educação-SBIE), volume 1.

Larkin-Hein, T. e Zollman, D. A. (2000). Digital video, learning styles, and student understanding of kinematics graphs. Journal of SMET Education, 1(2):4-17.

Loureiro, A. e Messias, I. (2016). Competences and learning profiles of digital age's students. In Handbook of Research on Engaging Digital Natives in Higher Education Settings, pages 171-191. IGI Global.

Luthra, P. e Mackenzie, S. (2020). 4 ways covid-19 could change how we educate future generations.

Martins, E. e Gouveia, L. (2019). Ml-sai: Um modelo pedagógico para atividades de m-learning que integra a abordagem da sala de aula invertida. In Anais do XXVII Workshop sobre Educação em Computação, pages 121-130. SBC.

Massey, A. P., Brown, S. A., e Johnston, J. D. (2005). It's all fun and games... until students learn. Journal of Information Systems Education, 16(1):9.

Mitchell, A., Petter, S., e Harris, A. (2017). Learning by doing: Twenty successful active learning exercises for information systems courses. Journal of Information Technology Education: Innovations in Practice, 16(1):21-46.

Tapscott, D. e Williams, A. D. (2010). Innovating the 21st-century university: It's time. Educause review, 45(1):16-29.

Zainuddin, Z. e Halili, S. H. (2016). Flipped classroom research and trends from different fields of study. International review of research in open and distributed learning, 17(3):313-340. 